\title{
The Intrapleural Bridge Connection is One of the Reasons for Unknown Localized Pleural Adhesion
}

\author{
Qihua $\mathrm{Gu}^{1,2}$ \\ Xinhao Deng ${ }^{1,2}$ \\ Zhao $\mathrm{Li}^{1,2}$ \\ Jing Wang ${ }^{3}$ \\ Chengping $\mathrm{Hu}^{1,2}$ \\ Shuhua Lei ${ }^{1,2}$ \\ Xiaoling Cai ${ }^{1,2}$ \\ 'Department of Respiratory Medicine, \\ Xiangya Hospital Affiliated to Central \\ South University, Changsha, Hunan \\ Province, 410008, People's Republic of \\ China; ${ }^{2}$ Key Cite of National Clinical \\ Research Center for Respiratory \\ Disease, Changsha, Hunan Province, \\ 410008, People's Republic of China; \\ ${ }^{3}$ Department of Pathology, Xiangya \\ Hospital Affiliated to Central South \\ University, Changsha, Hunan Province, \\ 410008, People's Republic of China
}

Correspondence: Qihua Gu

Tel +86 I5973196025

Email guqh06@I63.com
Background: Simple signs of local pleural adhesion are often found in people during a physical examination. In the present study, we aimed to clarify whether the merely localized pleural adhesion was just caused by previous pleural inflammation or physiological variation.

Materials and Methods: Chest X-ray image materials were collected to analyze the incidence of simple pleural adhesions. Moreover, the causes of these simple pleural adhesions were further analyzed using thoracoscopy under direct vision and biopsy data.

Results: In all 2218 chest X-ray images, 68 cases were found to have pleural lesions (3.07\%), including 15 cases of localized pleural adhesion only. Subsequently, we analyzed the characteristics of 70 cases of pleural lesions using thoracoscopy. In two lung cancer patients with pleural metastasis, we found an unusual pleural junction. This connective strip was smooth and free of inflammation, resembling the normal pleura.

Conclusion: Some of these purely localized pleural adhesions might be attributed to previous inflammation. However, there was still at least a possibility that there must be a physiological pleural junction, which could be the cause of the purely localized pleural adhesion shown in the chest radiograph.

Keywords: pleural adhesion, bridge connection, physiological variation, image, thoracoscope

\section{Introduction}

The parietal pleura adheres to the inner surface of the thoracic wall. The visceral pleura attaches to the surface of the lung and heart. In general, there is no restriction on the relative movement of these two types of pleurae. Once the visceral pleura adheres to the parietal pleura, the relative movement between the two pleurae is limited, In clinical practice, pleural adhesion is often detected using X-ray chest photographs and chest CT imaging. ${ }^{1-5}$ Sometimes, the pleural junction is detected by ultrasound. ${ }^{6,7}$ When adhesion between the parietal pleura and visceral pleura is presented, abnormal lung sliding, as well as the contraction and expansion of the lung, is restricted, leading to tension. Abnormal image signs, such as abnormal lung-sliding, pleural plaques, pleural traction, and pleural depression, are presented in CT images and ultrasonography. ${ }^{6-8}$

In terms of the etiology, the majority of pleural adhesions are caused by a pleural disease. Tuberculous pleurisy, ${ }^{9,10}$ malignant pleural effusion, ${ }^{11,12}$ invasion of lung cancer, ${ }^{4,13}$ rheumatoid immunological associated diseases ${ }^{14-16}$ and so on can lead to pleural adhesion. Lesions of pulmonary carcinoma invasion and membrane tumor proliferation result in fusion and fixation of the visceral pleura with the 
parietal pleura. Visible components, such as fibrin and cells, are presented in the pleural effusion induced by pleural inflammation and carcinomas. These visible components are deposited in the pleura to thicken the pleura. High levels of fibrin in the pleural inflammatory and tumor-induced exudates result in net adhesions, leading to adhesion between the visceral and parietal pleurae. The pleural lesions caused by pleurisy are probably still there after the patients have healed from pleural effusion, and it will be revealed on $\mathrm{CT}$ imaging and ultrasound visualization. ${ }^{17,18}$ However, we sometimes find localized pleural adhesions in chest X-rays or chest CT scans in individuals without any related symptoms. There is also no medical history of ever pleural disease in these individuals. In the present study, we aimed to elucidate these abnormal adhesions.

\section{Materials and Methods}

This study was carried out in two parts. First, chest X-ray images from healthy individuals were analyzed. Subsequently, pleural lesions in patients undergoing thoracoscopy were assessed. This study was conducted following the Declaration of Helsinki. We confirmed that the potential benefit to the patients outweighed the risk. The study was approved by the Medical Ethics Board of Xiangya Hospital, Affiliated to Central South University (No. 201,703,133). Each patient signed the informed consent and patient records/information was anonymized.

\section{Survey of X-Ray Chest Radiographs}

To investigate the incidence of local pleural adhesions in the "healthy" individuals, we enrolled people who had a scheduled annual physical examination in this survey. The volunteers received chest X-ray examination and left position radiography. The conventional chest X-ray images were analyzed by back-front and lateral positions. Lung lesions, costophrenic-angle abnormalities, and pleural adhesions were recorded in detail. All of the chest X-ray diagnoses were confirmed by two radiologists. The medical histories of all volunteers were questioned and recorded, and chest pain, dyspnea, and other symptoms related to pleural diseases were emphasized.

\section{Thoracoscopic Analysis}

We could not perform thoracoscopic surgery on patients whose images showed partial pleural connection due to ethical reasons. However, medical thoracoscopy is a commonly used method to diagnose and treat pleural effusion. We used the opportunity of clinical thoracoscopic treatment to find evidence of the physical pleural connection. Patients enrolled for pleural lesion analysis were patients undergoing thoracoscopic diagnosis and treatment. A total of 70 patients with pleural effusion underwent thoracoscopy, including 62 cases of exudative pleural effusion and eight cases of bloody pleural effusion. All of the 70 patients exhibited indications and no contraindication of thoracoscopy. The age of the patients ranged from 21 to 83, with an average age of $58 \pm 9$ years old. All 70 patients had shortness of breath after walking, climbing upstairs, daily labor work, and other activities. Chest pain and/or discomfort were found in 48 patients, coughs were found in 39 patients, and only 11 patients had a recent fever and night sweats. On the day of thoracoscopy, pleural effusion was drained, and an air volume of $600 \sim 800 \mathrm{~mL}$ was injected into the pleural cavity. X-ray chest radiography was performed before thoracoscopy. The 5th or 6th intercostal space on the front costal axillary was selected as the operative point referring to the chest radiograph. The skin of the patient was routinely disinfected with the iodine complex. Lidocaine was used for local anesthesia. Once a time, lidocaine solution containing $200 \mathrm{mg}$ lidocaine was extracted with a syringe and injected into the skin to form a local swelling firstly. Then subcutaneous and muscular layers were injected layer by layer, finally to the parietal pleura, and the anesthesia was enough before the operation. After the air could be pumped back, the remaining lidocaine was injected into the pleural cavity. An incision was made on the skin and subcutaneous tissue using a scalpel. A hemostatic forceps was used to separate the muscular layer and parietal pleura. The pleural effusion was further extracted through thoracoscopy after the thoracoscope entered the chest cavity. The parietal pleura, visceral pleura, septal pleura, mediastinal pleura, top of the pleura, and costophrenic angle were carefully examined using electron thoracoscopy. The major lesions in the pleura, as well as secondary lesions, were closely examined. A pleural biopsy was performed, and the diagnosis was made by two pathologists.

\section{Statistical Analysis}

Data were statistically analyzed with SPSS 13.0 software. The positive rate of pleural lesions between females and males, as well as the incidence of symptoms in patients with different pleural lesions, was evaluated by Crosstabs 
$\left(\chi^{2}\right.$ test $)$. A $\mathrm{P}$ value $<0.05$ was considered statistically significant.

\section{Results}

\section{X-Ray Imaging of Pleural Lesions}

We investigated pleural adhesion from people who underwent a physical examination by chest X-ray exam. Among 2218 individuals, 68 cases were found to have pleural lesions, reaching a positive rate of $3.07 \%$. The difference in the positive rate of pleural lesions between males and females was not statistically significant (Table 1). Pleural lesions could be roughly divided into three groups as follows: (A) pleural adhesion with pulmonary lesions, (B) pleural adhesion with a blunt costophrenic angle, and (C) localized pleural adhesion (Figure 1). Most of the subjects in the group (A) and group (B) had ever symptoms of chest pain, breathing difficulty, and a long-term ( $\geq 1$ week) of coughing. In group (C), the majority had no history of these above-mentioned symptoms. The differences in the incidence of symptoms among groups were statistically significant (Table 2).

\section{Pleural Lesions Diagnosed by Thoracoscopy}

Thoracoscopy can not be performed on subjects with a local pleural adhesion (healthy subjects) due to medical ethics rules. To clarify whether the merely localized pleural adhesion was just caused by previous pleural inflammation, we looked for evidence from patients who received thoracoscopy. In this study, we diagnosed 70 patients with pleural effusion by thoracoscopic pleural biopsy, including 40 cases of pleural metastasis of lung cancer, three cases of malignant pleural mesothelioma, 25 cases of tuberculous pleurisy, one case of purulent pleurisy, and one case of pleural amyloidosis. We analyzed the characteristics of 70 cases with pleural lesions under thoracoscopy. In some cases, there were two types of lesions on the pleura, including the primary pathological changes and secondary lesions. In two special patients, we found an unusual pleural junction. The patients had pleural

Table I Incidence of Pleural Lesions in a Physical Examination

\begin{tabular}{|l|l|l|l|}
\hline Group & Number & Pleural Lesion & Incidence (\%) \\
\hline Male & 1035 & 31 & 2.995 \\
Female & 1183 & 37 & $3.128^{*}$ \\
\hline
\end{tabular}

Note: $* \mathrm{P}>0.05$. effusion. Through minimally invasive thoracoscopic surgery, we found that the main lesions on their pleura were pleural masses and nodules, and other secondary lesions were simple pleural junctions, which were located between the visceral pleura and parietal pleura of the posterior costal region. The connective strips were smooth and free of inflammation, resembling the normal pleura (Figure 2).

\section{Discussion}

Diseases of the pleura and pleural cavity are common and significantly contribute to the workload of respiratory physicians. ${ }^{19,20}$ Pleural adhesion is often diagnosed with $\mathrm{X}$-ray chest photography. In the group of individuals who were examined on a chest X-ray, 68 cases of 2218 (3.07\%) individuals had pleural adhesions. More importantly, the actual incidence of pleural adhesion might be higher than that of the detected result. In individuals, the pulmonary pleura contracts with the expansion of the lung, and it can move freely with the parietal pleura. Pleural adhesion is most likely to be detected by X-ray chest radiography in the part of the pleura with the greatest lung physiological mobility, such as the diaphragmatic pleura. CT scans can reveal local pleural adhesions in other areas (Figure 1), while chest X-ray radiography is difficult to expose these pleural adhesions. Moreover, it will be more helpful in detecting local pleural adhesions by using softwareassisted analysis on dynamic-ventilation computed tomography compared with chest static CT scans. ${ }^{21}$ However, in the present study, we focused on a merely localized pleural adhesion (Figure 1). Local adhesion of diaphragmatic pleura was found in $0.68 \%$ of individuals who received a physical examination, showing a triangularshaped pulmonic pull sign between the base of the lung and the diaphragm. Unlike another pleural adhesion where the intercostal septum is blunt, this type of pleural adhesion had a smooth periphery without lung lesions and a sharp intercostal diaphragm. Furthermore, this pleural adhesion between the base of the lung and the diaphragm was found in people without pleurisy, pleural effusion, or tumors. Based on the features of this particular pleural adhesion and no history of the disease, it was suggested that this pleural adhesion was not caused by pleural disease. According to the characteristics of this special pleural adhesion in pleural imaging, and the individuals had no disease history, suggesting that this pleural adhesion was not caused by pleural disease, while it was a physiological variation. If this particular pleural adhesion 


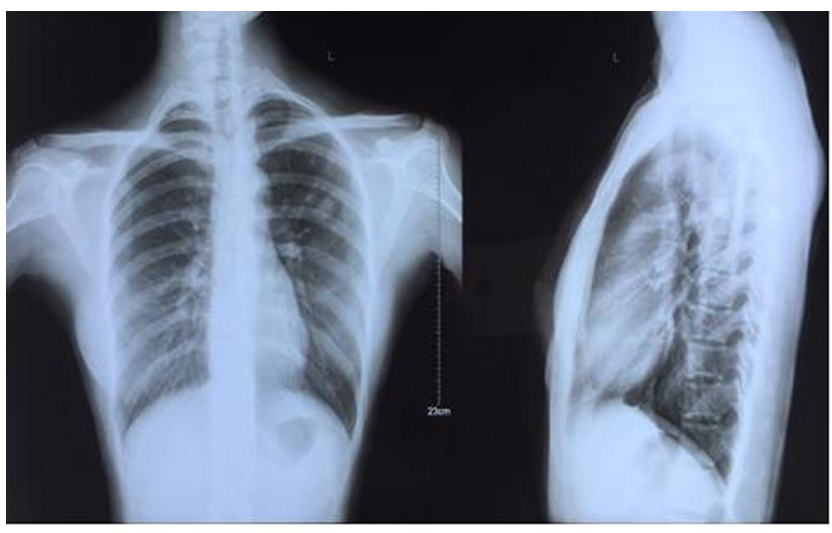

A

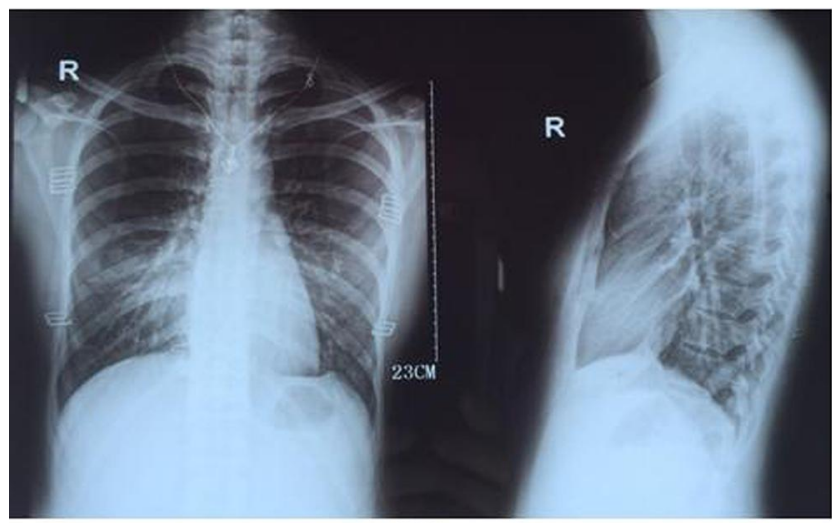

C

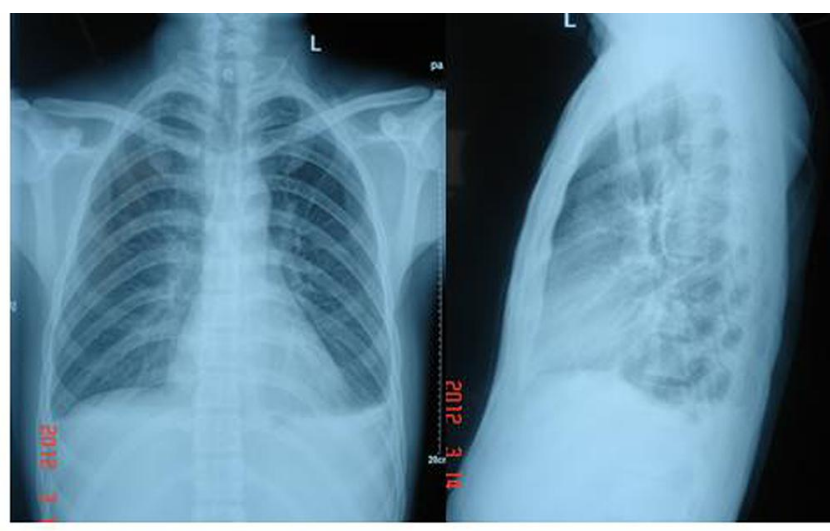

B
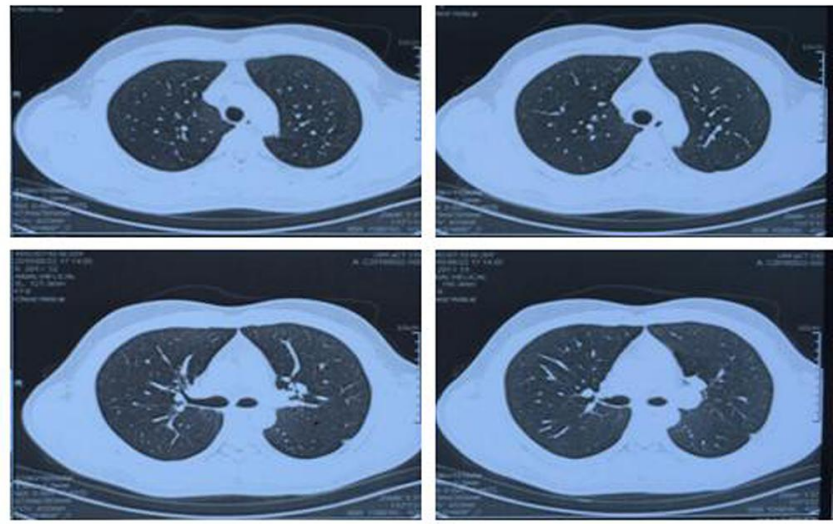

D

Figure I Chest X-ray examination. (A) Pleural adhesion with pulmonary lesions. (B) Pleural adhesion with a blunt costophrenic angle. (C) Localized pleural adhesion only. (D) CT scan shows a localized pleural adhesion.

was merely a physiological variation, it was difficult to replicate with animal models. These people can not be identified by surgical biopsy because of ethical constraints. We came up with the idea that thoracoscopic biopsy is the most reliable way to diagnose the cause of pleural disease. ${ }^{22-25}$ Therefore, we attempted to get evidence with the help of thoracoscopy. We performed a pleural examination during electronic medical thoracoscopy on patients with exudative pleural effusion. In addition to analyzing the etiology of pleural effusion, special attention should be paid to what may not be related to the cause of the pleural effusion. With two thoracoscopic procedures,

Table 2 Incidence of Different Symptoms of Pleural Lesions

\begin{tabular}{|l|l|l|l|}
\hline Group & A** & B** & C** \\
\hline Number & 24 & 29 & 15 \\
Ever symptoms & 17 & 15 & $3^{*}$ \\
\hline
\end{tabular}

Notes: ${ }^{*} \mathrm{P}<0.05$. $*$ A A pleural adhesion with pulmonary lesions. B: pleural adhesion with blunt a costophrenic angle. C: localized pleural adhesion. we found an intrapleural bridge connection. The patients were found with nodular lesions on the pleura and diagnosed as carcinoma by biopsy. We found a junction band between the posterior costal parietal pleura and the visceral pleura. The connective band was smooth, which was like the normal pleura, and it was diagnosed as pleural tissue by histopathology. This finding confirmed our hypothesis that there was a bridge connection between the parietal pleura and visceral pleura. The physiological variation of pleural junction might explain, at least partially, the unexplained local pleural adhesion.

The improved knowledge of pleural physiology can help the clinicians in clinical decision making, as well as the diagnosis and treatment of pleural disease. ${ }^{26}$ There was no evidence that this physiological pleural junction was innate or acquired. The pleurae are formed as a result of an intricate genetic regulation during development. The lung develops at about 4-6 weeks of gestation in humans. ${ }^{27}$ The pleural cavity is created between the 4th and 7th week of 


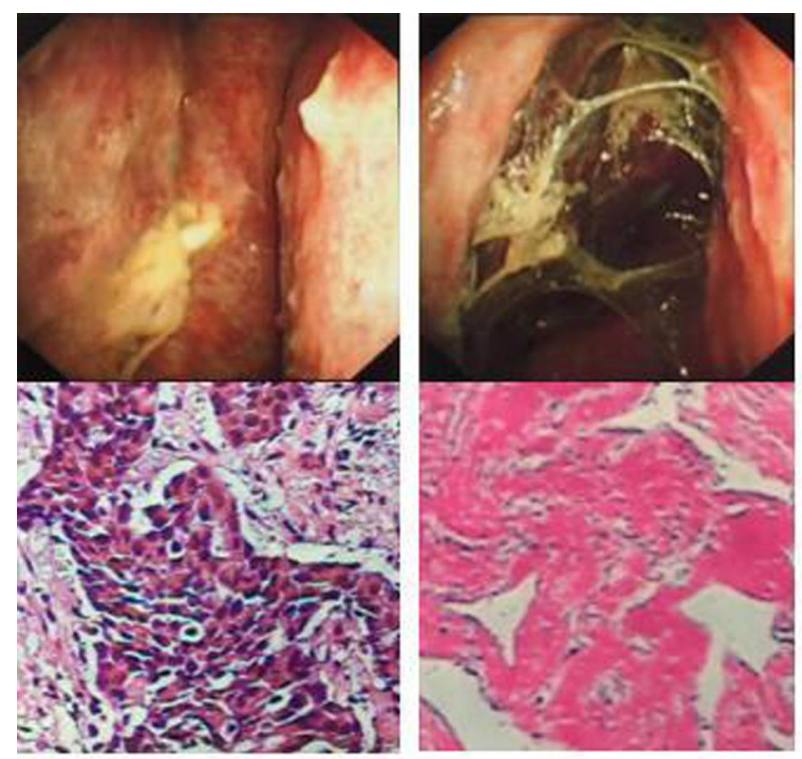

A

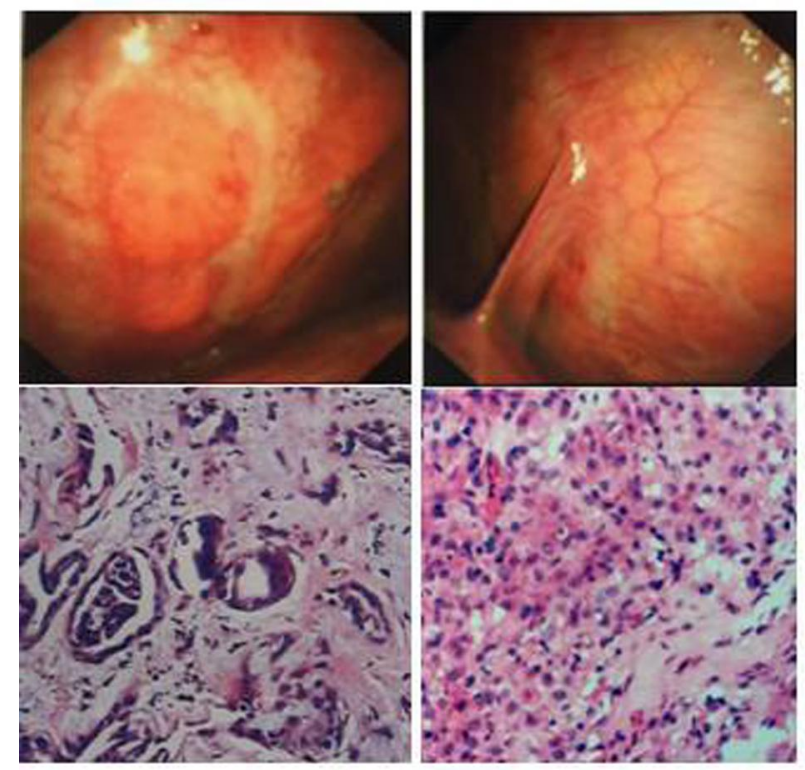

C
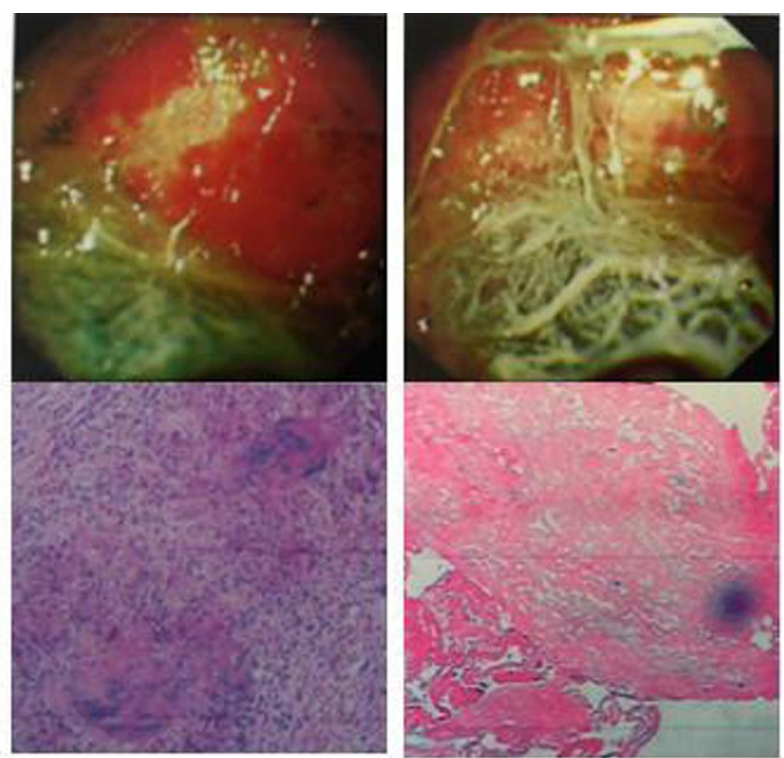

B
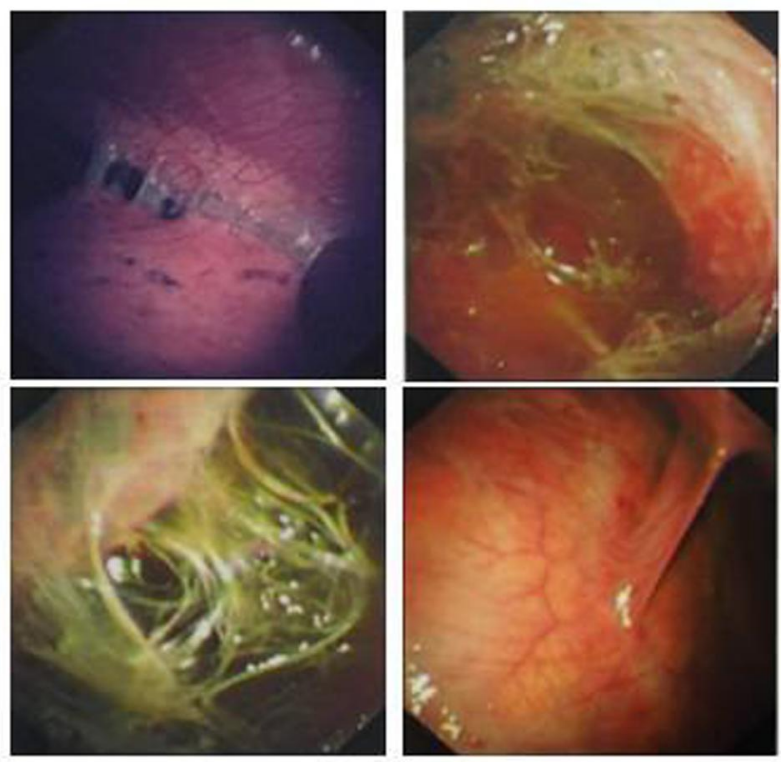

D

Figure 2 Thoracoscopic view of the pleural changes and biopsy. (A) The manly lesions were pleural thickening, uneven surfaces, and nodules on the pleura, and the histopathological diagnosis was adenocarcinoma. Secondary pleural lesions were pleural adhesions with obvious adhesion band formation, and biopsy demonstrated fibrinoid degeneration. (B) The main pleural lesions were extensive pleural congestion, swelling, and thickening, and the biopsy was diagnosed as tuberculous pleurisy. The pleural showed secondary lesions of extensive pleural adhesion with obvious adhesion band formation, and biopsy demonstrated fibrinoid degeneration. (C) The main pleural lesions were pleural masses and nodules, and the biopsy diagnosis was adenocarcinoma. It was also found that there was a junction band between the parietal pleura and visceral pleura. This junction band was very similar to the normal pleura. Biopsy showed fibrous tissue, mesenchymal cells, and adipose tissue. (D) Pleural adhesion caused by pleural diseases was a common adhesion between visceral pleura and parietal pleura, which was not smooth, fragile, and easily be broken. Unlike pleural adhesion caused by pleural disease, physiological pleural variation was an extension of normal pleural tissue that was easily recognized.

embryologic development, and it is lined by the splanchnopleura and somatopleurae. ${ }^{28}$ The pulmonary pleurae are thin at 9-16 weeks of gestation, and a smooth lung surface is established largely by the thick submesothelial tissue, including veins and lymphatic vessels, until 26 weeks. ${ }^{29}$ After this stage, the visceral pleurae are separated from the parietal pleurae. Therefore, the local undivided incidence of pulmonary pleura and somatic pleura is most likely to occur between the 6th and 26th weeks of embryologic development due to abnormal pleural development. Previous studies have shown that pleural mesothelial cells undergo mesothelial-mesenchymal transition and 
migrate into the lungs. ${ }^{30,31}$ However, it is unlikely that the pneumopleural adhesion will be physiologically fused with the parietal pleura again after birth. In this study, we had no clear evidence that such particular pleural junction was congenital or postnatal. However, there was at least a possibility that there must be a physiological pleural junction, which could be the cause of the purely localized pleural adhesion shown in the chest radiograph.

\section{Data Sharing Statement}

All data generated or analyzed during this study are available from the corresponding author Qihua Gu upon reasonable request.

\section{Ethics Approval and Consent to Participate}

This study was conducted in accordance with the Declaration of Helsinki. The experiment was approved by the Ethics Department of Xiangya Hospital, Central South University and each patient signed the informed consent.

\section{Acknowledgment}

The authors thank the Bronchoscope Room of Respiratory Medicine in the Xiangya Hospital affiliated to the Central South University, Department of Pathology in Xiangya Hospital affiliated to the Central South University, Department of Radiology of Xiangya Hospital affiliated to the Central South University for their support.

\section{Author Contributions}

Qihua Gu, Xinhao Deng are co-first authors. All authors made a significant contribution to the work reported, whether that is in the conception, study design, execution, acquisition of data, analysis and interpretation, or in all these areas; took part in drafting, revising or critically reviewing the article; gave final approval of the version to be published; have agreed on the journal to which the article has been submitted; and agree to be accountable for all aspects of the work.

\section{Funding}

This work was supported by the National Key R\&D Program of China (Grant No 2016YFC1303800).

\section{Disclosure}

The authors declare that they have no conflict of interest.

\section{References}

1. van Geffen WH, Klooster K, Hartman JE, et al. Pleural adhesion assessment as a predictor for pneumothorax after endobronchial valve treatment. Respiration. 2017;94(2):224-231. doi:10.1159/000477258

2. Mason AC, Miller BH, Krasna MJ, White CS. Accuracy of CT for the detection of pleural adhesions: correlation with video-assisted thoracoscopic surgery. Chest. 1999;115(2):423-427. doi:10.1378/ chest.115.2.423

3. Hashimoto M, Nagatani Y, Oshio Y, et al., investigators of ACTIve study group. Preoperative assessment of pleural adhesion by Four-Dimensional Ultra-Low-Dose Computed Tomography (4D-ULDCT) with Adaptive Iterative Dose Reduction using Three-Dimensional processing (AIDR-3D). Eur $J$ Radiol. 2018;98:179-186. doi:10.1016/j.ejrad.2017.11.011

4. Sakuma K, Yamashiro T, Moriya H, Murayama S, Ito H. Parietal pleural invasion/adhesion of subpleural lung cancer: quantitative 4-dimensional CT analysis using dynamic-ventilatory scanning. Eur J Radiol. 2017;87:36-44. doi:10.1016/j.ejrad.2016.12.004

5. Tokuno J, Shoji T, Sumitomo R, Ueda Y, Yamanashi K, Huang C-L. Preoperative detection of pleural adhesions by respiratory dynamic computed tomography. World J Surg Oncol. 2017;15(1):212. doi:10.1186/s12957-017-1280-7

6. Cassanelli N, Caroli G, Dolci G, et al. Accuracy of transthoracic ultrasound for the detection of pleural adhesions. Eur J Cardiothorac Surg. 2012;42(5):813-818. doi:10.1093/ejcts/ezs144

7. Uemura A, Fukayama T, Tanaka T, Tanaka R. Utility of lung ultrasonography for detection of pleural adhesions in dogs. $J$ Ultrasound Med. 2018;37(5):1193-1198. doi:10.1002/jum.14468

8. Amelon RE, Cao K, Reinhardt JM, Christensen GE, Raghavan ML. A measure for characterizing sliding on lung boundaries. Ann Biomed Eng. 2014;42(3):642-650. doi:10.1007/s10439-013-0920-5

9. Shaw JA, Irusen EM, Diacon AH, Koegelenberg CF. Pleural tuberculosis: a concise clinical review. Clin Respir J. 2018;12 (5):1779-1786. doi:10.1111/crj.12900

10. Zhou S, Zhao J, Song X, Zheng M, Li H, Pan Y. Imaging manifestations of B-mode ultrasound combined with CT in tuberculous pleuritis patients and the diagnostic value. Exp Ther Med. 2018;16 (3):2343-2348. doi:10.3892/etm.2018.6471

11. Gu Q, Hu C, Qu J. Good response of malignant pleural effusion from carcinoma of unknown primary site to the anti-tuberculosis therapy: a case report. Int J Clin Exp Pathol. 2013;6(5):973-977.

12. Vakil E, Ost D, Vial MR, et al. Non-specific pleuritis in patients with active malignancy. Respirology. 2018;23(2):213-219. doi:10.1111/ resp. 13187

13. Hanaoka T, Sone S, Takayama F, Hayano T, Yamaguchi S, Okada M. Presence of local pleural adhesion in CT screening-detected small nodule in the lung periphery suggests noncancerous, inflammatory nature of the lesion. Clin Imaging. 2007;31(6):385-389. doi:10.1016/ j.clinimag.2007.04.029

14. Ryu S, Fu W, Petri MA. Associates and predictors of pleurisy or pericarditis in SLE. Lupus Sci Med. 2017;4(1):1-9. doi:10.1136/ lupus-2017-000221

15. Nagayasu A, Kubo S, Nakano K, et al. IgG4-related pleuritis with elevated adenosine deaminase in pleural effusion. Intern Med. 2018;57(15):2251-2257. doi:10.2169/internalmedicine.0387-17

16. Kawaguchi H, Tsuboi H, Yagishita M, et al. Severe adult-onset still disease with constrictive pericarditis and pleuritis that was successfully treated with tocilizumab in addition to corticosteroids and cyclosporin A. Intern Med. 2018;57(7):1033-1038. doi:10.2169/ internalmedicine.9809-17

17. de Fonseka D, Edey A, Stadon L, Viner J, Darby M, Maskell NA. The physiological consequences of different distributions of diffuse pleural thickening on CT imaging. $B r \quad J$ Radiol. 2017;90 (1077):20170218. doi:10.1259/bjr.20170218 
18. Halaweh T, Adkins E, King A. Ultrasound visualization of a pleural adhesion. J Emerg Trauma Shock. 2015;8(3):167-168. doi:10.4103/ 0974-2700.160731

19. Carlucci P, Trigiani M, Mori PA, et al. Competence in pleural procedures. Panminerva Med. 2018. doi:10.23736/S00310808.18.03564-3564

20. Khan JA, Lehtomäki AI, Toikkanen VJ, Ukkonen MT, Nevalainen RM, Laurikka JO. Long-term prognosis and causes of death after pleural infections. Scand J Surg. 2018;107(2):145-151. doi:10.1177/1457496917738868

21. Nagatani Y, Hashimoto M, Oshio Y, et al; investigators of ACTIve study group. Preoperative assessment of localized pleural adhesion: utility of software-assisted analysis on dynamic-ventilation computed tomography. Eur J Radiol. 2020;133:109347. doi:10.1016/j. ejrad.2020.109347

22. Munavvar M, Khan MA, Edwards J, Waqaruddin Z, Mills J. The autoclavable semirigid thoracoscope: the way forward in pleural disease? Eur Respir J. 2007;29(3):571-574. doi:10.1183/ 09031936.00101706

23. Gao BA, Zhou G, Guan L, Zhang LY, Xiang GM. Effectiveness and safety of diagnostic flexi-rigid thoracoscopy in differentiating exudative pleural effusion of unknown etiology: a retrospective study of 215 patients. J Thorac Dis. 2014;6(5):438-443. doi:10.3978/j. issn.2072-1439.2014.02.09

24. Yang Y, Wu YB, Wang Z, et al. Long-term outcome of patients with nonspecific pleurisy at medical thoracoscopy. Respir Med. 2017;124:1-5. doi:10.1016/j.rmed.2017.01.005
25. McDonald CM, Pierre C, de Perrot M, et al. Efficacy and cost of awake thoracoscopy and video-assisted thoracoscopic surgery in the undiagnosed pleural effusion. Ann Thorac Surg. 2018;106 (2):361-367. doi:10.1016/j.athoracsur.2018.02.044

26. Akulian J, Yarmus L, Feller-Kopman D. The evaluation and clinical application of pleural physiology. Clin Chest Med. 2013;34(1):11-19. doi:10.1016/j.ccm.2012.11.001

27. Warburton D. Overview of lung development in the newborn human. Neonatology. 2017;111(4):398-401. doi:10.1159/000458465

28. Lee KF, Olak J. Anatomy and physiology of the pleural space. Chest Surg Clin N Am. 1994;4(3):391-403.

29. Yamamoto M, Wilting J, Abe H, Murakami G, Rodríguez-Vázquez JF, Abe SI. Development of the pulmonary pleura with special reference to the lung surface morphology: a study using human fetuses. Anat Cell Biol. 2018;51(3):150-157. doi:10.5115/ acb.2018.51.3.150

30. Batra H, Antony VB. The pleural mesothelium in development and disease. Front Physiol. 2014;5:284. doi:10.3389/fphys.2014.00284

31. Ysasi AB, Wagner WL, Valenzuela CD, et al. Evidence for pleural epithelial-mesenchymal transition in murine compensatory lung growth. PLoS One. 2017;12(5):e0177921. doi:10.1371/journal. pone. 0177921
International Journal of General Medicine

\section{Publish your work in this journal}

The International Journal of General Medicine is an international, peer-reviewed open-access journal that focuses on general and internal medicine, pathogenesis, epidemiology, diagnosis, monitoring and treatment protocols. The journal is characterized by the rapid reporting of reviews, original research and clinical studies
Dovepress

across all disease areas. The manuscript management system is completely online and includes a very quick and fair peer-review system, which is all easy to use. Visit http://www.dovepress.com/ testimonials.php to read real quotes from published authors. 